\title{
Keratoprosthesis prophylaxis: is it time for a paradigm shift?
}

This article was published in the following Dove Press journal: Clinical Ophthalmology

\author{
JS Pelletier ${ }^{1-3}$ \\ SB Barone' \\ JA Capriotii ${ }^{1,2}$ \\ 'Department of Ophthalmology, \\ Veloce BioPharma LLC, Fort \\ Lauderdale, FL, USA; ${ }^{2}$ Department \\ of Ophthalmology, Plessen \\ Ophthalmology Consultants, \\ Christiansted, VI, USA; ${ }^{3}$ Department \\ of Ophthalmology, Ocean \\ Ophthalmology Group, Miami, FL, USA
}

\begin{abstract}
The Boston Type I Keratoprosthesis has been improving in both design and safety since its inception. Due to particular features inherent in the Boston Type I Keratoprosthesis eye and certain aspects of the ocular surface, special attention is required to maintain these implanted devices. There is currently a prominent role for keratoprosthesis prophylaxis; it is designed to prevent infectious complications like keratitis and endophthalmitis. This standard-of-care therapy has anecdotally been shown to improve outcomes; however, it has not been examined in the setting of controlled clinical trials. Moreover, concerns remain with the chronic utilization of topical antibiotics in that they may engender antibiotic resistance and select for opportunistic populations to establish a foothold on the ocular surface. We believe and introduce the idea that there is merit in exploring other compounds besides antibiotics for prophylaxis such as antiseptics like povidone-iodine. Specifically developed formulations of povidone-iodine may prove useful in both improving keratoprosthesis safety and simultaneously mitigating concerns regarding antibiotic resistance.
\end{abstract}

Keywords: keratoprosthesis, ocular surface, povidone-iodine, antimicrobial resistance

\section{Background}

The Boston Type I Keratoprosthesis (KPro) is indicated to restore vision in a variety of pathologic corneal conditions which contribute to visual loss on a global scale. ${ }^{1}$ This well-engineered medical device, conceived in the 19th century and in modern development since the mid 20th, is the most commonly implanted KPro and is generally reserved for cases where corneal transplants are projected to fail due to advanced ocular comorbidities. It is fashioned in a collar-button configuration with a front plate and optical stem made of poly-(methyl methacrylate) and a snap-on titanium backplate which compresses the host cornea during assembly. ${ }^{2,3}$ Despite early difficulty in device retention and safety, outcomes in KPro eyes have improved due to enhanced material biocompatibility and a better understanding of the biological and environmental factors affecting the health of the ocular surface and anterior segment. ${ }^{4}$ While there are a variety of complications inherent in the procedure, two vexing and potentially avoidable complications include endophthalmitis and keratitis. ${ }^{5}$ Studies have demonstrated variable rates of these infections ranging from $0 \%$ to $11 \%$ and $0 \%$ to $17 \%$, respectively. ${ }^{6,7}$

\section{Purpose}

The severity of infectious endophthalmitis in this setting cannot be understated as it can "virtually destroy the eye overnight." "A key contributing anatomic concept is the notion that the KPro is the only implanted ocular device that offers a direct communication 
from the ocular surface to the sterile aspects of the inner eye. Because the ocular surface is known to be colonized by heterogeneous populations of microorganisms, it follows that any potential breach in this system may predispose to a sight-threatening infection via a transmigration of microbes. Utilizing high-resolution corneal ocular coherence tomography, it is possible to identify defects in epithelial sealing between the KPro and the host cornea which are most likely to manifest as "gaps" at the optical stem-host junction. ${ }^{9}$ Not only are such microscopic anatomic variables at play, but there are also a number of other risk factors that contribute to the development of infectious keratitis. They include but are not limited to poor biointegration of the device, chronic steroid use, poor functioning of the innate immune system of the ocular surface, chronic inflammation around the prosthesis, and persistent epithelial defects. ${ }^{10,11}$ Whether an eye develops keratitis or endophthalmitis might depend on the location of the initial infection and its proximity to the deeper vitreous cavity. For instance, an infection that develops rapidly and is in proximity to the KPro stem may more easily slide into other compartments of the eye. ${ }^{6}$ In the 1990 s, it was realized that to better stabilize the ocular surface, cover persistent epithelial defects, and prevent corneal melting, bandage contact lenses should be routinely employed. While this protocol improved KPro outcomes, one cannot dispute the association of this practice with Gram-negative and fungal keratitis. ${ }^{12}$

In light of these findings, it is logical that a standard of care developed which called for KPro patients to utilize longterm topical antibiotics in the hope of mitigating infectious sequelae. While not formally studied in prospective, randomized, doubly-masked clinical trials, this practice has appeared, in anecdotal studies and series, to significantly improve outcomes and patient safety. Current guidelines published by the Massachusetts Eye and Ear Infirmary call for the usage of low-dose topical formulations of fourth-generation fluoroquinolones (FQ) or trimethoprim/polymyxin for standard cases and the addition of topical vancomycin for those deemed to be high risk. ${ }^{13}$ Despite this, there is often significant variation and at times disagreement in both antibiotic choice and dosing frequency among corneal surgeons. Moreover, looming over this practice are the concerns implicit in the lifelong utilization of topical antibiotics. It is well established that antibiotics can contribute to antimicrobial resistance, alter the ocular microbiota, alter the microbiota at extraocular sites, and enhance the potential for fungal infection. In order to better understand the purview of these effects on the ocular surface flora, a more granular view with respect to selection pressure and resistance is instructive.

In a landmark study conducted in 2011, eyes receiving chronic antibiotics after intravitreal injection demonstrated a marked increase in antibiotic resistance at both the level of the conjunctiva and the nasopharyngeal mucosa. ${ }^{14}$ The downstream dangers of antimicrobial resistance were also highlighted in this report. Specifically, the presence of antibiotic-resistant organisms in the nasopharynx was shown to increase susceptibility to systemic infections like pneumonia. A similar study published in 2012 corroborated these findings and reported in a subset of data that $87.5 \%$ of eyes were resistant to FQ after being exposed to topical FQ therapy for 4 days after each intravitreal injection. ${ }^{15}$ One year later, a study on microbial resistance patterns present in KPro eyes was published. In this manuscript, the ocular flora was identified in the KPro eye and the colonizing bacterial species were found to be similar to those colonizing the healthy eye, predominantly Staphylococcus epidermidis and other coagulase-negative Staphylococcus species. The level of identifiable FQ resistance among the KPro group in this study was rather alarming. ${ }^{3}$ Other reports have also raised the issue of fungal and Gram-negative infections in the KPro eyes. With respect to the former, it is thought that the use of topical steroids and antibiotics may render the eye susceptible to colonization and infection with these undesirable microbes. ${ }^{16}$ In further support of this, the literature reports that fungal etiologies are ascendant and responsible for approximately $10 \%$ of all cases of KPro related endophthalmitis. ${ }^{17}$ Finally, chronically dosed antibiotics like vancomycin that specifically target Gram-positive species may select for more robust and opportunistic Gram-negative colonies to outcompete and establish an ocular surface stronghold. This has been reflected in increasing reported rates of Gram-negative endophthalmitis in KPro patients. ${ }^{18}$

\section{Conclusion}

Given this body of data, it is compelling to consider other agents outside of traditional antibiotics for infectious prophylaxis in KPro eyes. It is clear that this subset is at risk for visual loss due to compromised ocular anatomy and immunologic function which facilitates microbes, both traditionally benign and/or opportunistic, to establish virulence. An ideal agent would thwart this risk by being polymicrobial in nature, efficacious against biofilms, nontoxic, unable to promote resistance, and ideally dosed QD to maintain compliance. There is understandably some hesitation to test new therapeutic candidates, for the withholding of 
current therapy, however imperfect, may present an ethical dilemma. Nonetheless, some early work to this effect has been carried out with hypochlorous acid and potential further investigation appears promising. ${ }^{19}$ The authors of this manuscript believe the answer might lie in further exploration of povidone-iodine(PVP-I). Existing PVP-I ophthalmic formulations $(5 \% \mathrm{~W} / \mathrm{w}$ in aqueous solvent) have already been indicated for at-risk KPro patients and we believe PVP-I therapy fulfills many of the compulsory criteria of the ideal single agent. ${ }^{20}$ Cornerstone to this belief is the notion that PVP-I is an effective biocidal agent that has not been shown to engender antimicrobial resistance. ${ }^{21,22}$ Multiple in vitro studies support the prodigious ability of PVP-I formulations to eliminate bacteria, fungi, yeast, viruses, and even acanthamoeba. ${ }^{23-26}$ Furthermore, it has been reported that PVP-I is able to reduce bacterial load and colony counts on the conjunctival surface prior to ocular surgery and that this effect does not alter the constituents which comprise the normal conjunctival flora. ${ }^{22,27,28}$ It should be understood that a reduction of bacterial load, but not bacterial diversity, would be unlikely to promulgate the growth of more virulent bacterial strains. Another important attribute of PVP-I is its ability to destroy the bacterial biofilm. ${ }^{29}$ This eluted extracellular matrix is vital to the survival of microbes when under constant assault from the host immune system. Although the role of biofilms in KPro is currently being elucidated, they have been positively identified under electron microscopy in patients who have undergone explantation. Research in this area supports the notion that biofilms should be a prime therapeutic target as not only do they play a conventional role in conferring antimicrobial resistance and contributing to keratitis/endophthalmitis but are also implicated in chronic inflammation, which may predispose to corneal melts, retroprosthetic membranes, and macular edema. ${ }^{30}$

Given the body of evidence in support of PVP-I, the ratelimiting aspect of its development is less likely to be efficacy related, but perhaps lies in its tolerability and potential ocular surface toxicity. There are reports by the current authors and others of novel, dilute PVP-I formulations for ophthalmic use which appear to mitigate these concerns. ${ }^{31-34}$ By carefully modifying the solvent vehicle, $\mathrm{pH}$ and available iodine concentration we believe that an optimal formulation can be produced and incorporated into clinical trials. We believe these specifically developed formulations could be useful in order to lessen the burden of postoperative infection and prevent the virulent transformation of the ocular surface without promoting antibiotic resistance during chronic, long-term use.

\section{Author contributions}

All authors contributed to data analysis, drafting and revising the article, gave final approval of the version to be published, and agree to be accountable for all aspects of the work.

\section{Disclosure}

All authors declare the financial interest that they own stock and/or are employees of Veloce BioPharma and may receive reimbursements, fees, funding, or salary from the organization that may in any way gain or lose financially from the publication of the manuscript, either now or in the future. The authors report no other conflicts of interest in this work.

\section{References}

1. Oliva MS, Schottman T, Gulati M. Turning the tide of corneal blindness. Indian J Ophthalmol. 2012;60(5):423-427.

2. Harissi-Dagher M, Khan BF, Schaumberg DA, Dohlman CH. Importance of nutrition to corneal grafts when used as a carrier of the Boston Keratoprosthesis. Cornea. 2007;26(5):564-568.

3. Dohlman $\mathrm{CH}$, Harissi-Dagher M, Khan BF, et al. Introduction to the use of the Boston keratoprosthesis. Expert Rev Ophthalmol. 2006; 1(1):41-48.

4. Salvador-Culla B, Kolovou PE. Keratoprosthesis: A Review of Recent Advances in the Field. J Funct Biomater. 2016;7(2):13.

5. Wagoner MD, Welder JD, Goins KM, Greiner MA. Microbial Keratitis and Endophthalmitis After the Boston Type 1 Keratoprosthesis. Cornea. 2016;35(4):486-493.

6. Robert MC, Eid EP, Saint-Antoine P, Harissi-Dagher M. Microbial colonization and antibacterial resistance patterns after Boston type 1 keratoprosthesis. Ophthalmology. 2013;120(8):1521-1528.

7. Nouri M, Terada H, Alfonso EC, et al. Endophthalmitis after keratoprosthesis: incidence, bacterial causes, and risk factors. Arch Ophthalmol. 2001;119(4):484-489.

8. Behlau I, Martin KV, Martin JN, et al. Infectious endophthalmitis in Boston keratoprosthesis: incidence and prevention. Acta Ophthalmol. 2014;92(7):e546-e555.

9. Zarei-Ghanavati S, Betancurt C, Mas AM, Wang J, Perez VL. Ultra high resolution optical coherence tomography in Boston type I keratoprosthesis. J Ophthalmic Vis Res. 2015;10(1):26-32.

10. Chan CC, Holland EJ. Infectious keratitis after Boston type 1 keratoprosthesis implantation. Cornea. 2012;31(10):1128-1134.

11. Sejpal K, Yu F, Aldave AJ. The Boston keratoprosthesis in the management of corneal limbal stem cell deficiency. Cornea. 2011;30(11): 1187-1194.

12. Barnes SD, Dohlman CH, Durand ML. Fungal colonization and infection in Boston keratoprosthesis. Cornea. 2007;26(1):9-15.

13. Harvard Medical School. Recommendations Boston KPro Follow-up. Boston, MA: Harvard Medical School. Available from: https://eye. hms.harvard.edu/eyeinsights/2015-september/recommendations-forboston-kpro-follow-up. Accessed September 10, 2018.

14. Kim SJ, Toma HS. Ophthalmic antibiotics and antimicrobial resistance a randomized, controlled study of patients undergoing intravitreal injections. Ophthalmology. 2011;118(7):1358-1363.

15. Milder E, vander J, Shah C, Garg S. Changes in antibiotic resistance patterns of conjunctival flora due to repeated use of topical antibiotics after intravitreal injection. Ophthalmology. 2012;119(7):1420-1424.

16. Barnes SD, Dohlman CH, Durand ML. Fungal colonization and infection in Boston keratoprosthesis. Cornea. 2007;26(1):9-15.

17. Chan CC, Holland EJ. Infectious endophthalmitis after Boston type 1 keratoprosthesis implantation. Cornea. 2012;31(4):346-349. 
18. Greiner MA, Li JY, Mannis MJ. Longer-term vision outcomes and complications with the Boston type 1 keratoprosthesis at the University of California, Davis. Ophthalmology. 2011;118(8):1543-1550.

19. Odorcic S, Haas W, Gilmore MS, Dohlman CH. Fungal Infections After Boston Type 1 Keratoprosthesis Implantation: Literature Review and In Vitro Antifungal Activity of Hypochlorous Acid. Cornea. 2015;34(12): 1599-1605.

20. Magalhães FP, do Nascimento HM, Ecker DJ, et al. Microbiota evaluation of patients with a Boston type I keratoprosthesis treated with topical $0.5 \%$ moxifloxacin and 5\% povidone-iodine. Cornea. 2013;32(4):407-411.

21. Lanker Klossner B, Widmer HR, Frey F. Nondevelopment of resistance by bacteria during hospital use of povidone-iodine. Dermatology. 1997;195(Suppl 2):10-13.

22. Hsu J, Gerstenblith AT, Garg SJ, vander JF. Conjunctival flora antibiotic resistance patterns after serial intravitreal injections without postinjection topical antibiotics. Am J Ophthalmol. 2014;157(3):514-518.

23. Grzybowski A, Kanclerz P, Myers WG. The use of povidone-iodine in ophthalmology. Curr Opin Ophthalmol. 2018;29(1):19-32.

24. Goldenheim PD. In vitro efficacy of povidone-iodine solution and cream against methicillin-resistant Staphylococcus aureus. Postgrad Med J. 1993;69(Supp1 3):S62-S65.

25. Traoré O, Fayard SF, Laveran H. An in-vitro evaluation of the activity of povidone-iodine against nosocomial bacterial strains. J Hosp Infect. 1996;34(3):217-222.

26. Wada H, Nojima Y, Ogawa S, et al. Relationship between Virucidal Efficacy and Free Iodine Concentration of Povidone-Iodine in Buffer Solution. Biocontrol Sci. 2016;21(1):21-27.
27. Panahibazaz M, Moosavian M, Khataminia G, et al. Sub-Conjunctival Injection of Antibiotics vs. Povidone-Iodine Drop on Bacterial Colonies in Phacoemulsification Cataract Surgery. Jundishapur J Microbiol. 2014;7(9):e13108.

28. González Bandrés C, Carrilero Ferrer MJ, Buznego Suárez L, et al. Efficacy of topical povidone-iodine applied the day before cataract surgery to reduce conjunctival flora. Arch Soc Esp Oftalmol. 2001; 76(4):229-234.

29. Hoekstra MJ, Westgate SJ, Mueller S. Povidone-iodine ointment demonstrates in vitro efficacy against biofilm formation. Int Wound J. 2017;14(1):172-179.

30. Sivaraman KR, Hou JH, Chang JH, et al. Scanning Electron Microscopic Analysis of Biofilm Formation in Explanted Human Boston Type I Keratoprostheses. Cornea. 2016;35(1):25-29.

31. Pelletier JS, Capriotti KD, Stewart KS, Barone SB, Capriotti JA. A novel transdermal ophthalmic preparation for blepharitis in a dilute povidone-iodine and dimethyl sulfoxide (DMSO) system: a case series. EC Ophthalmology. 2018;9(3):129-134.

32. Pelletier JS, Stewart KP, Capriotti K, Capriotti JA. Rosacea Blepharoconjunctivitis Treated with a Novel Preparation of Dilute Povidone Iodine and Dimethylsulfoxide: a Case Report and Review of the Literature. Ophthalmol Ther. 2015;4(2):143-150.

33. Pelletier JS, Capriotti K, Stewart KS, Capriotti JA. Demodex blepharitis treated with a novel dilute povidone-iodine and DMSO System: a case report. Ophthalmol Ther. 2017;6(2):361-366.

34. Pelletier JS, Stewart K, Trattler W, et al. A combination povidone-iodine $0.4 \%$ /dexamethasone $0.1 \%$ ophthalmic suspension in the treatment of adenoviral conjunctivitis. Adv Ther. 2009;26(8):776-783.
Clinical Ophthalmology

\section{Publish your work in this journal}

Clinical Ophthalmology is an international, peer-reviewed journal covering all subspecialties within ophthalmology. Key topics include: Optometry; Visual science; Pharmacology and drug therapy in eye diseases; Basic Sciences; Primary and Secondary eye care; Patient Safety and Quality of Care Improvements. This journal is indexed on Submit your manuscript here: http://www.dovepress.com/clinical-ophthalmology-journal

\section{Dovepress}

PubMed Central and CAS, and is the official journal of The Society of Clinical Ophthalmology (SCO). The manuscript management system is completely online and includes a very quick and fair peer-review system, which is all easy to use. Visit http://www.dovepress.com/ testimonials.php to read real quotes from published authors. 